UWThPh-1999-61

December 1999

\title{
Two-Particle Correlations and Meson-Antimeson Mixing Effects
}

\author{
G.V. Dass \\ Physics Department, Indian Institute of Technology \\ Powai, Bombay - 400076 , India \\ W. Grimus \\ Institut für Theoretische Physik, Universität Wien \\ Boltzmanngasse 5, A-1090 Vienna, Austria
}

\begin{abstract}
We discuss 2-particle correlations which arise in the time evolution of C-odd and C-even meson-antimeson states of flavoured neutral mesons. In order to keep our discussion general, we do not use the Weisskopf - Wigner approximation. Possible deviations from quantum-mechanical coherence effects are parameterized by a so-called decoherence parameter $\zeta$. In particular, we study the $\zeta$-dependence of the asymmetry of unlike and like-flavoured events which was recently observed experimentally in the $K^{0} \bar{K}^{0}$ system. In this $\zeta$ dependence, we point out some important general features which do not rely on the Weisskopf - Wigner approximation. Some other related results are derived more generally than in the literature.
\end{abstract}

PACS numbers: 03.65.Bz, 13.20.Eb, 13.25.Hw 


\section{INTRODUCTION}

Quantum-mechanical (QM) correlations arising in the time evolution of the 2-particle wave functions $|f \otimes \bar{f}-\bar{f} \otimes f\rangle$, where $f$ is some flavoured neutral meson like $f=K^{0}, B_{d}^{0}$ and $\bar{f}$ is its antiparticle, have been studied recently (see Refs. [1] and papers cited therein). The aim in these papers was to use data in order to see whether the correlations expected on the basis of quantum mechanics were present in full, partial or zero strength. To achieve this, a so-called "decoherence parameter" $\zeta$ was introduced [5]. The conclusions have been in favour of "full strength", as far as the present data can say. The usual phenomenology of the $(f, \bar{f})$ complex has been utilized in the various analyses. This phenomenology is based on the Weisskopf-Wigner approximation (WWA) [6].

Because of the importance of the conclusions, it is advisable to make the analysis as model-independent as possible. The purpose of the present note is to see how far one may go without involving the WWA. By the same token, the numerical predictive power is, of course, greatly reduced if the WWA is not invoked. The WWA is considered in this paper only for comparison with the general results which we derive. We shall make some corresponding remarks on the time evolution of the state $|f \otimes \bar{f}+\bar{f} \otimes f\rangle$ also. In addition, some related results will be derived more generally than in the literature.

The plan of the paper is as follows. In Sec. II, we give the basic framework required for discussing the time evolution of the two-particle states $|f \otimes \bar{f} \pm \bar{f} \otimes f\rangle$ in the general and various special cases. Sec. III is devoted to the evaluation of the recently observed experimental asymmetry [7] (see also the relevant experimental papers cited in Refs. [2,3]) between the rates of the production of like-flavoured (viz. $|f \otimes f\rangle$ and $|\bar{f} \otimes \bar{f}\rangle$ ) and unlikeflavoured (viz. $|f \otimes \bar{f}\rangle$ and $|\bar{f} \otimes f\rangle$ ) states of the $(f, \bar{f})$ complex. In Sec. IV, we note that some purely QM results hold more generally than the derivations in the literature; this includes also cases within the WWA. Sec. V is devoted to a systematic discussion of the basis needed for a vanishing of the experimental asymmetry under the Furry hypothesis (absence of QM interference terms, corresponding to $\zeta=1$ ] [8]; we show that the conclusion of Ref. [1] needs generalization. In Sec. VI, we try to see how the introduction of $\zeta$ effectively induces a CP violation; we also study a special configuration considered in Ref. [1]. Our derivation is more general and much simpler than in the literature.

\section{THE FORMALISM}

Starting from the states $|f\rangle$ and $|\bar{f}\rangle$ initially, i.e., at proper time $t=0$, the time evolution is given by [9, 10]

$$
\begin{aligned}
& |f\rangle \stackrel{t}{\rightarrow} a(t)|f\rangle+b(t)|\bar{f}\rangle+\sum_{i} e_{i}(t)\left|\rho_{i}\right\rangle, \\
& |\bar{f}\rangle \stackrel{t}{\rightarrow} \bar{b}(t)|f\rangle+\bar{a}(t)|\bar{f}\rangle+\sum_{i} \bar{e}_{i}(t)\left|\rho_{i}\right\rangle,
\end{aligned}
$$

where the $\left|\rho_{i}\right\rangle(i=1,2, \ldots)$ are other states, orthogonal to $|f\rangle$ and $|\bar{f}\rangle$, produced with coefficients $e_{i}$ and $\bar{e}_{i}$. We shall need only the amplitudes $a, \bar{a}, b$ and $\bar{b}$. While $a$ and $\bar{a}$ denote the diagonal transitions $|f\rangle \rightarrow|f\rangle$ and $|\bar{f}\rangle \rightarrow|\bar{f}\rangle$, respectively, $b$ and $\bar{b}$ denote the 
"mixing" or non-diagonal transitions $|f\rangle \rightarrow|\bar{f}\rangle$ and $|\bar{f}\rangle \rightarrow|f\rangle$, respectively. We shall keep the amplitudes $a, \bar{a}, b$ and $\bar{b}$ unknown and independent, unless otherwise stated.

The WWA provides a model for these four amplitudes. Note that by definition [10] we have

$$
a(0)=\bar{a}(0)=1, \quad b(0)=\bar{b}(0)=0, \quad e_{i}(0)=\bar{e}_{i}(0)=0 \quad \forall i
$$

Taking $|\bar{f}\rangle=\mathcal{C P}|f\rangle, \mathrm{CP}$ violation means that $a \neq \bar{a}$ and/or $b \neq \bar{b}$. It is useful to introduce the CP-odd amplitudes

$$
v=\frac{1}{2}(a-\bar{a}), \quad V=\frac{1}{2}(b-\bar{b})
$$

and the CP-even amplitudes

$$
A=\frac{1}{2}(a+\bar{a}), \quad B=\frac{1}{2}(b+\bar{b}) .
$$

Then, $v=0$ corresponds to CPT (and CP) invariance, and $V=0$ to $\mathrm{CP}$ (and T) invariance.

The $(f, \bar{f})$ complex may be equally well described in terms of the normalized superpositions

$$
\begin{aligned}
& \left|f_{1}\right\rangle=p_{1}|f\rangle+q_{1}|\bar{f}\rangle,\left|p_{1}\right|^{2}+\left|q_{1}\right|^{2}=1, \\
& \left|f_{2}\right\rangle=p_{2}|f\rangle-q_{2}|\bar{f}\rangle,\left|p_{2}\right|^{2}+\left|q_{2}\right|^{2}=1
\end{aligned}
$$

where $p_{1,2}$ and $q_{1,2}$ are some complex constants. Exploiting the freedom of the three unmeasurable phases, one may use the Eberhard phase convention [11] to write

$$
\begin{aligned}
& p_{1}=e^{i \theta / 2} \cos \alpha_{1}, \quad q_{1}=e^{-i \theta / 2} \sin \alpha_{1}, \\
& p_{2}=e^{-i \theta / 2} \cos \alpha_{2}, \quad q_{2}=e^{i \theta / 2} \sin \alpha_{2} .
\end{aligned}
$$

Note that the ranges of these parameters can be confined to $0 \leq \alpha_{1,2} \leq \pi / 2$ and $-\pi / 2 \leq$ $\theta \leq \pi / 2$ without loss of generality. Instead of $\alpha_{1}$ and $\alpha_{2}$, one can also use the angles $\sigma, \delta$ defined as

$$
\sigma=\frac{\pi}{2}-\left(\alpha_{1}+\alpha_{2}\right), \quad \delta=\alpha_{1}-\alpha_{2}
$$

Thus the three significant parameters occurring in Eq.(2.5) can be taken to be the real CP-violating parameters $\theta, \sigma$ and $\delta$. The scalar product of the states in Eq.(2.5) can be expressed as

$$
\left\langle f_{2} \mid f_{1}\right\rangle=\sin \sigma \cos \theta+i \cos \delta \sin \theta
$$

As a corollary, the CP eigenstates

$$
\left|f_{ \pm}\right\rangle=\frac{1}{\sqrt{2}}(|f\rangle \pm|\bar{f}\rangle)
$$

arise with the choice 


$$
\theta=\sigma=\delta=0
$$

corresponding to

$$
\alpha_{1,2}=\frac{\pi}{4} \quad \text { and } \quad p_{1,2}=q_{1,2}=\frac{1}{\sqrt{2}}
$$

The WWA is characterized by the introduction of independently propagating states $\left|f_{S, L}\right\rangle$ which are superpositions (with constant complex coefficients $p_{S, L}$ and $q_{S, L}$ ) of $|f\rangle$ and $|\bar{f}\rangle$ :

$$
\begin{aligned}
& \left|f_{S}\right\rangle=p_{S}|f\rangle+q_{S}|\bar{f}\rangle, \quad\left|p_{S}\right|^{2}+\left|q_{S}\right|^{2}=1 \\
& \left|f_{L}\right\rangle=p_{L}|f\rangle-q_{L}|\bar{f}\rangle, \quad\left|p_{L}\right|^{2}+\left|q_{L}\right|^{2}=1 .
\end{aligned}
$$

These states have the time evolution

$$
\left|f_{S, L}\right\rangle \stackrel{t}{\rightarrow} \Theta_{S, L}(t)\left|f_{S, L}\right\rangle
$$

with

$$
\Theta_{S, L}(t)=\exp \left\{-i t\left(m_{S, L}-\frac{i}{2} \Gamma_{S, L}\right)\right\},
$$

where $m_{S, L}$ and $\Gamma_{S, L}$ are the usual masses and widths for $\left|f_{S, L}\right\rangle$. There are only two definite $t$-dependences now, given by $\Theta_{S, L}(t)$. The initial conditions (2.2) imply that $v, V$ and $B$ must have the $t$-dependence $\left[\Theta_{S}(t)-\Theta_{L}(t)\right]$. Indeed, one finds (see Ref. [12] for a review)

$$
\begin{aligned}
a+\bar{a} & =\Theta_{S}+\Theta_{L}, \\
a-\bar{a} & =\left(\frac{p_{S}}{q_{S}}-\frac{p_{L}}{q_{L}}\right) b, \\
\bar{b} & =\left(\frac{p_{S} p_{L}}{q_{S} q_{L}}\right) b, \\
b & =\frac{q_{S} q_{L}}{p_{S} q_{L}+p_{L} q_{S}}\left(\Theta_{S}-\Theta_{L}\right) .
\end{aligned}
$$

Also, Eqs.(2.16) and (2.17) imply the relations

$$
v(t)=c_{1} B(t) \quad \text { and } \quad V(t)=c_{2} B(t)
$$

with constants $c_{1,2}$ being given by the relations (2.16) and (2.17). The two relations in Eq.(2.19) can be conceived as "general" consequences of the WWA, while the stronger relations (2.15) and (2.18) are necessary for explicit calculations using the WWA fully. In Eq.(2.19) we note that

$$
\begin{array}{r}
\mathrm{CPT}(\mathrm{CP}) \text { invariance } \Rightarrow c_{1}=0, \\
\mathrm{CP}(\mathrm{T}) \text { invariance } \Rightarrow c_{2}=0 .
\end{array}
$$

Note that the $c_{1,2}$ are defined in the WWA, but not in general. 
The above 1-particle description is required in the analysis of the time evolution of the 2-particle states

$$
|f \otimes \bar{f}+\epsilon \bar{f} \otimes f\rangle,
$$

where $\epsilon=+1$ and -1 denote the C-even and C-odd states, respectively. Examples of $\epsilon=-1$ are the $\phi$ meson, the $\Upsilon(4 \mathrm{~S})$ and the ${ }^{3} \mathrm{~S}_{1}$ state of $p \bar{p}$ annihilation at rest [7]. An example of $\epsilon=+1$ would be the state $|s\rangle$ obtained by radiative decays of $\Upsilon(4 \mathrm{~S})$ :

$$
\Upsilon(4 \mathrm{~S}) \rightarrow s+\gamma .
$$

Denoting the proper times involved in the time evolution of the states (2.22) by $t^{\prime}$ and $t$ and using the notation

$$
a^{\prime} \equiv a\left(t^{\prime}\right), \quad a \equiv a(t), \quad \ldots,
$$

we can write the time evolution in terms of these eight amplitudes which, in general, are all unknown [10].

Different bases $\left(f_{1}, f_{2}\right)$ (2.5) used in expressing the $t^{\prime}$, $t$-evolution of (2.22) correspond to different $p_{1,2}$ and $q_{1,2}$. One can introduce the $t$-dependent amplitudes $A_{1,2}$ and $\bar{A}_{1,2}$ as

$$
\left|f_{1,2}\right\rangle \stackrel{t}{\rightarrow} A_{1,2}|f\rangle+\bar{A}_{1,2}|\bar{f}\rangle
$$

with

$$
\left(\begin{array}{l}
A_{1} \\
A_{2}
\end{array}\right)=\left(\begin{array}{rr}
p_{1} & q_{1} \\
p_{2} & -q_{2}
\end{array}\right)\left(\begin{array}{l}
a \\
\bar{b}
\end{array}\right), \quad\left(\begin{array}{l}
\bar{A}_{1} \\
\bar{A}_{2}
\end{array}\right)=\left(\begin{array}{rr}
p_{1} & q_{1} \\
p_{2} & -q_{2}
\end{array}\right)\left(\begin{array}{l}
b \\
\bar{a}
\end{array}\right) .
$$

The choice $p_{1}=-q_{2}=1, q_{1}=p_{2}=0$ would correspond to the $(f, \bar{f})$ basis. The notation (2.24) indicating the association of the primed amplitudes with $t^{\prime}$ and of the unprimed amplitudes with $t$ will apply also to $A_{1,2}$ and $\bar{A}_{1,2}$. Possible CP invariance of amplitudes refers to $a, \bar{a}, b$ and $\bar{b}$, viz. $v=0$ and/or $V=0$. On the other hand, the general mixing parameters lead to the basis provided by the CP eigenstates $\left|f_{ \pm}\right\rangle$of Eq.(2.9), if the choice (2.11) is made. If, however, one specializes to the WWA, the amplitudes and the mixing parameters $\left(p_{S, L} ; q_{S, L}\right)$ are intimately related; the CP property of the amplitudes is given by the choice (2.20) and (2.21) of these mixing parameters.

Special cases of the completely general amplitudes could be (i) the general CP-invariant amplitudes $(v=V=0)$ without the WWA, (ii) the WWA amplitudes, which may also obey $\mathrm{CP}$ invariance $\left(c_{1}=c_{2}=0\right)$. Similarly, the special cases of the general basis states $\left|f_{1,2}\right\rangle$ could be the $\left|f_{ \pm}\right\rangle$, or the flavour states $|f\rangle,|\bar{f}\rangle$, or the WWA states $\left|f_{S, L}\right\rangle$ characterized by independent propagation. Of course, one can also have $\left|f_{S, L}\right\rangle$ becoming $\left|f_{ \pm}\right\rangle$as a further assumption.

\section{EVALUATION OF THE EXPERIMENTALLY OBSERVED ASYMMETRY IN THE GENERAL AND SPECIAL CASES}

The experimental observable recently investigated [1, 4,0$]$ utilizes the production of the like-flavoured states $|f \otimes f\rangle$ and $|\bar{f} \otimes \bar{f}\rangle$ and the unlike-flavoured states $|f \otimes \bar{f}\rangle$ and $|\bar{f} \otimes f\rangle$. 
We use $t^{\prime}$ for the time of the measurement of the first meson and $t$ for the second meson. The CP-even observable [7] is the asymmetry $\mathcal{A}\left(t^{\prime}, t\right)$ between the numbers of all like-flavoured (viz. $f f$ and $\bar{f} \bar{f}$ ) events and all unlike-flavoured (viz. $f \bar{f}$ and $\bar{f} f$ ) events:

$$
\mathcal{A}\left(t^{\prime}, t\right)=\frac{\mathcal{N}_{\text {unlike }}\left(t^{\prime}, t\right)-\mathcal{N}_{\text {like }}\left(t^{\prime}, t\right)}{\mathcal{N}_{\text {unlike }}\left(t^{\prime}, t\right)+\mathcal{N}_{\text {like }}\left(t^{\prime}, t\right)} .
$$

We first concentrate on the state provided by the choice $\epsilon=-1$ in Eq.(2.22). This $\mathrm{CP}$-odd state can be rewritten in terms of the general basis (2.5). Taking into account the time evolution, one simply gets

$$
\left|f_{1}\left(t^{\prime}\right) \otimes f_{2}(t)-f_{2}\left(t^{\prime}\right) \otimes f_{1}(t)\right\rangle
$$

where, for the channels relevant to (3.1),

$$
\left|f_{1,2}(t)\right\rangle=A_{1,2}(t)|f\rangle+\bar{A}_{1,2}(t)|\bar{f}\rangle,
$$

and, similarly, for the argument $t^{\prime}$. Thus, for the calculation of $\mathcal{A}\left(t^{\prime}, t\right)$ we use Eq.(2.25) for the time evolution and multiply the interference term by the factor $(1-\zeta)$ in order to parameterize deviations from quantum mechanics [5, 1] [4. This leads to the general expression

$$
\mathcal{A}\left(t^{\prime}, t\right)=\frac{N_{1}\left(t^{\prime}, t\right)+2(1-\zeta) D_{1}\left(t^{\prime}, t\right)}{N_{2}\left(t^{\prime}, t\right)+2(1-\zeta) D_{2}\left(t^{\prime}, t\right)}
$$

where

$$
\begin{aligned}
& N_{1,2}=\left[\left|A_{2}^{\prime}\right|^{2} \mp\left|\bar{A}_{2}^{\prime}\right|^{2}\right]\left[\left|\bar{A}_{1}\right|^{2} \mp\left|A_{1}\right|^{2}\right]+\left(t \leftrightarrow t^{\prime}\right), \\
& D_{1,2}=\operatorname{Re}\left\{\left[-A_{2}^{\prime *} A_{1}^{\prime} \pm \bar{A}_{2}^{* *} \bar{A}_{1}^{\prime}\right]\left[\bar{A}_{1}^{*} \bar{A}_{2} \mp A_{1}^{*} A_{2}\right]\right\}
\end{aligned}
$$

Here, the real decoherence parameter $\zeta$ [5] is just one way of expressing possible departures from quantum mechanics. While quantum mechanics corresponds to $\zeta=0$, complete decoherence (Furry's hypothesis [8]) corresponds to $\zeta=1$, which means absence of the QM correlations expressed by the interference terms $D_{1,2}$ of Eq.(3.6).

In order to be able to consider the case of small departures from $\mathrm{CP}$ conservation (i.e., the basis states $\left|f_{1,2}\right\rangle$ are close to $\left|f_{ \pm}\right\rangle$and the CP-violating amplitudes $v$ and $V$ of Eq.(2.3) are small relative to $A$ and $B$ of Eq.(2.4)), we write the amplitudes $A_{1,2}$ and $\bar{A}_{1,2}$, up to first order in $\mathrm{CP}$ violation, as

$$
\begin{aligned}
& A_{1}=\frac{1}{\sqrt{2}}\left(A+B+\Delta_{1}\right), \\
& \bar{A}_{1}=\frac{1}{\sqrt{2}}\left(A+B-\Delta_{1}\right), \\
& A_{2}=\frac{1}{\sqrt{2}}\left(A-B+\Delta_{2}\right), \\
& \bar{A}_{2}=-\frac{1}{\sqrt{2}}\left(A-B-\Delta_{2}\right),
\end{aligned}
$$

with

$$
\Delta_{1}=v-V+\beta_{1}(A-B), \quad \Delta_{2}=v+V+\beta_{2}(A+B) .
$$


The quantities

$$
\begin{aligned}
& \frac{1}{\sqrt{2}} \beta_{1}=p_{1}-\frac{1}{\sqrt{2}}=\frac{1}{\sqrt{2}}-q_{1}=\frac{1}{2 \sqrt{2}}(\sigma+i \theta-\delta), \\
& \frac{1}{\sqrt{2}} \beta_{2}=p_{2}-\frac{1}{\sqrt{2}}=\frac{1}{\sqrt{2}}-q_{2}=\frac{1}{2 \sqrt{2}}(\sigma-i \theta+\delta)
\end{aligned}
$$

denote the first order CP-violating corrections for the coefficients $p_{1,2}, q_{1,2}$ of Eq.(2.5). Now it is easy to show that $N_{1}$ and $D_{2}$ are quantities of second order of smallness and that $N_{2}$ and $D_{1}$ have no contribution of first order of CP violation. Thus, neglecting effects of second order of CP violation, the asymmetry (3.4) becomes

$$
\left.\mathcal{A}\left(t^{\prime}, t\right)\right|_{\epsilon=-1}=(1-\zeta) \mathcal{A}_{\epsilon=-1}^{\mathrm{QM}},
$$

where

$$
\mathcal{A}_{\epsilon=-1}^{\mathrm{QM}}=\frac{2 \operatorname{Re}\left(A_{1}^{\prime *} A_{2}^{*} A_{2}^{\prime} A_{1}\right)}{\left|A_{1}^{\prime} A_{2}\right|^{2}+\left|A_{2}^{\prime} A_{1}\right|^{2}}
$$

is the QM asymmetry neglecting second order of CP violation. Note that, for Eq.(3.11), we effectively have

$$
A_{1}=\bar{A}_{1}=\frac{1}{\sqrt{2}}(A+B), \quad A_{2}=-\bar{A}_{2}=\frac{1}{\sqrt{2}}(A-B) .
$$

The proportionality of $\mathcal{A}\left(t^{\prime}, t\right)$ to $(1-\zeta)$, apart from terms of second order in CP violation, has been noted in Ref. [1] in explicit calculations using the WWA fully and assuming CP conservation in mixing. Our derivation shows that the result does not depend on the WWA.

Coming now to the case $\epsilon=+1$ and rewriting the state (2.22) in terms of the general basis (2.5), we obtain

$$
\left|2 p_{2} q_{2} f_{1} \otimes f_{1}-2 p_{1} q_{1} f_{2} \otimes f_{2}+\left(p_{2} q_{1}-p_{1} q_{2}\right)\left(f_{1} \otimes f_{2}+f_{2} \otimes f_{1}\right)\right\rangle,
$$

where we have neglected an irrelevant normalization factor. If we proceed now by replacing the states $\left|f_{1,2}\right\rangle$ by the time-evolved states as in Eq.(3.2), we see that there are several possibilities to introduce decoherence, in contrast to Eq.(3.2). However, there is a drastic simplification if we assume CP invariance: $p_{1} q_{1}=p_{2} q_{2}=1 / 2$ and $p_{2} q_{1}-p_{1} q_{2}=0$. Then, if we assume $\mathrm{CP}$ invariance, our starting point for $\epsilon=+1$ is given by

$$
\left|f_{+}\left(t^{\prime}\right) \otimes f_{+}(t)-f_{-}\left(t^{\prime}\right) \otimes f_{-}(t)\right\rangle
$$

analogous to the state (3.2). In analogy to Eq.(3.10), we obtain

$$
\left.\mathcal{A}\left(t^{\prime}, t\right)\right|_{\epsilon=+1}=(1-\zeta) \mathcal{A}_{\epsilon=+1}^{\mathrm{QM}},
$$

where

$$
\mathcal{A}_{\epsilon=+1}^{\mathrm{QM}}=\frac{2 \operatorname{Re}\left(A_{1}^{* *} A_{1}^{*} A_{2}^{\prime} A_{2}\right)}{\left|A_{1}^{\prime} A_{1}\right|^{2}+\left|A_{2}^{\prime} A_{2}\right|^{2}} .
$$

Thus, in the case of $\epsilon=+1$ also, the asymmetry (3.1) is proportional to $(1-\zeta)$ if $\mathrm{CP}$ invariance holds; the amplitudes in Eq.(3.16) are again given by Eq.(3.12). 
If the basis states are $|f\rangle$ and $|\bar{f}\rangle$, the introduction of the decoherence parameter proceeds in the same way for $\epsilon= \pm 1$, but the dependence of the asymmetry (3.1) on $\zeta$ is not so simple:

$$
\left.\mathcal{A}\left(t^{\prime}, t\right)\right|_{\epsilon= \pm 1}=\frac{\left(\left|a^{\prime}\right|^{2}-\left|b^{\prime}\right|^{2}\right)\left(|a|^{2}-|b|^{2}\right)+4 \epsilon(1-\zeta) \operatorname{Im}\left(a^{\prime *} b^{\prime}\right) \operatorname{Im}\left(b^{*} a\right)}{\left(\left|a^{\prime}\right|^{2}+\left|b^{\prime}\right|^{2}\right)\left(|a|^{2}+|b|^{2}\right)+4 \epsilon(1-\zeta) \operatorname{Re}\left(a^{\prime *} b^{\prime}\right) \operatorname{Re}\left(b^{*} a\right)}
$$

wherein the CP-violating amplitudes $v, V$ have been dropped. This $\zeta$-dependence and the non-zero value of the asymmetry for $\zeta=1$ have been noted in Ref. [1] (see also Refs. [2 [4) for $\epsilon=-1$, in explicit calculations using the WWA fully. Again, our derivation shows the result to be more general. In the case of the Furry hypothesis $(\zeta=1)$ the non-zero asymmetry for $\epsilon=+1$ is the same as for $\epsilon=-1$, as seen from Eq.(3.17). This is similar to the corresponding comparison between the asymmetries (both zero) using the basis states $\left|f_{ \pm}\right\rangle$(see Eqs.(3.10) and (3.15)).

\section{PURELY QUANTUM-MECHANICAL RESULTS}

It is easy to see that the basis independence (viz. $p_{1,2}$ and $q_{1,2}$ can be arbitrary) of the net QM amplitude, starting from Eq.(2.22), for any detected final states (which may be made up of states other than $|f\rangle$ and $|\bar{f}\rangle$ used in Section [II]) holds quite generally: (i) for $\epsilon=+1$ and $\epsilon=-1$, (ii) arbitrary transition amplitudes from $|f\rangle$ and $|\bar{f}\rangle$ to the detected final states. It is really a matter of transferring (2.22) to the $\left|f_{1,2}\right\rangle$ basis using the transformation inverse to that in Eq.(2.5). Then, upon introducing transition amplitudes, one applies the transformations analogous to Eq.(2.26) for the chosen final detected states. In the process, the $p_{1,2}$ and $q_{1,2}$ drop out and the overall transition amplitude is seen to be the same as in the $(f, \bar{f})$ basis, in general. This basis independence [1 4 has been noted for $\epsilon=-1$ and using the WWA.

As a corollary, one may note for $\zeta \neq 0$ (i.e., in the case of departure from quantum mechanics), the value of $\zeta$ inferred from experimental data is, in general, basis-dependent. Because the QM amplitude is basis-independent, the corresponding probability $P_{\mathrm{QM}}$ is basisindependent as well. Writing

$$
P_{\mathrm{QM}}=P_{\text {Furry }}+P_{\text {int }},
$$

both $P_{\text {Furry }}$ and $P_{\text {int }}$ are, in general, basis-dependent (see, e.g., Eqs. 3.4 3.6)). Comparing the $\zeta$-modified version

$$
P_{\mathrm{QM}}=P_{\text {Furry }}+(1-\zeta) P_{\text {int }}
$$

of Eq.(4.1) with experimental data will lead to different numerical values for $\zeta$ for different bases because $P_{\text {int }}$ is basis-dependent. This has been noted in explicit calculations with the WWA in Refs. 迎田.

We now make some comments on QM calculations within the WWA, using Eq.(2.19) but not Eqs.(2.15, 2.18). Within this general WWA one gets [9,10,13]

$$
\frac{\mathcal{N}(f, f)}{\mathcal{N}(\bar{f}, \bar{f})}=\left(\frac{1-c_{2}}{1+c_{2}}\right)^{2}
$$


for $\epsilon=-1$. In this equation, $\mathcal{N}(\stackrel{(-)}{f}, \stackrel{(-)}{f})$ denotes the number of events yielding $|\stackrel{(-)}{f} \otimes \stackrel{(-)}{f}\rangle$. Thus, the two "like" states are produced in a proportion which is independent of $t^{\prime}$ and $t$, quite generally, within the WWA. The corresponding case of $\epsilon=+1$ requires the additional assumption of CPT invariance $\left(c_{1}=0\right)$, again with the result (4.3). If one requires the corresponding ratio for the "unlike" states to be constant for $\epsilon= \pm 1$, one obtains the constant to be unity for all $t^{\prime}$ and $t$, if CPT invariance $\left(c_{1}=0\right)$ holds:

$$
\frac{\mathcal{N}(f, \bar{f})}{\mathcal{N}(\bar{f}, f)}=1 .
$$

In all the above cases $c_{2} \neq 0$ is allowed. The results (4.3) and (4.4) for $\epsilon= \pm 1$ are to be compared with the simple results

$$
\frac{\mathcal{N}(f, f)}{\mathcal{N}(\bar{f}, \bar{f})}=\frac{\mathcal{N}(f, \bar{f})}{\mathcal{N}(\bar{f}, f)}=1
$$

at any $t^{\prime}, t$, if CP invariance of the amplitudes holds $(v=V=0)$, even without the WWA. Eq.(4.5) arises because the initial state is a $\mathrm{CP}$ eigenstate, and under $\mathrm{CP}$ one has

$$
|f \otimes f\rangle \rightarrow|\bar{f} \otimes \bar{f}\rangle \quad \text { and } \quad|f \otimes \bar{f}\rangle \rightarrow|\bar{f} \otimes f\rangle .
$$

While Eqs.(4.3) and (4.4) are based on the WWA without $V=0$ (in the form of $c_{2}=0$ ), Eq.(4.5) does not use the WWA, but it does use $V=0$. Eq.4.3) does not need CPT invariance (viz. $c_{1}=0$ ) for $\epsilon=-1$, but it does need $c_{1}=0$ for $\epsilon=+1$. The relation $c_{1}=0$ is also needed for Eq.(4.4). Some of the above results have been noted in explicit calculations using the WWA fully, namely Eqs.4.3) and (4.4) for the special choice $p_{S}=p_{L}$, $q_{S}=q_{L}$ and $\epsilon=-1,[1]$.

\section{VANISHING OF THE ASYMMETRY UNDER THE FURRY HYPOTHESIS}

For $\zeta=1$ one gets complete decoherence (the Furry hypothesis) and the interference terms characterizing 2-particle QM correlations disappear. If furthermore the basis is given by $\left|f_{ \pm}\right\rangle$and the amplitudes are CP-invariant, the asymmetry vanishes for both $\epsilon=+1$ and $\epsilon=-1$, [1] (see Eqs.(3.10) and (3.15)). One can ask [1]: What is the basis for which the asymmetry vanishes under the Furry hypothesis? Is it necessarily the $\left|f_{ \pm}\right\rangle$basis? We shall try to answer this question assuming CP invariance of the amplitudes $(v=V=0)$, but not the WWA. The case of general amplitudes (without CP invariance) can easily be seen to be pathological. Taking $\epsilon=-1$, one basically wants $N_{1}=0$, which can be written as

$$
G\left(t^{\prime}\right) F(t)+G(t) F\left(t^{\prime}\right)=0
$$

with the help of Eq.(3.5), using

$$
G(t)=\left|A_{2}\right|^{2}-\left|\bar{A}_{2}\right|^{2}, \quad F(t)=\left|\bar{A}_{1}\right|^{2}-\left|A_{1}\right|^{2} .
$$

Thus one wants 


$$
\begin{aligned}
G & =0 \text { if } \quad F \neq 0 \\
\text { or } \quad F & =0 \text { if } \quad G \neq 0 \\
\text { or } \quad F & =G=0 .
\end{aligned}
$$

To study the consequences, we note that Eq.(5.3) means

$$
\left(|a|^{2}-|b|^{2}\right)\left(\left|p_{2}\right|^{2}-\left|q_{2}\right|^{2}\right)+4 \operatorname{Im}\left(a^{*} b\right) \operatorname{Im}\left(p_{2}^{*} q_{2}\right)=0 .
$$

Because of the unmeasurable relative phase between $|f\rangle$ and $|\bar{f}\rangle$, the parameter $\operatorname{Im}\left(p_{2}^{*} q_{2}\right)$ can be chosen arbitrarily. Dropping it, Eq.(5.6) gives

$$
\left|p_{2}\right|=\left|q_{2}\right|
$$

because $|a|$ and $|b|$ are in general different. Therefore, with $\operatorname{Im}\left(p_{2}^{*} q_{2}\right)=0$, we have

$$
\left|f_{2}\right\rangle \rightarrow\left|f_{+}\right\rangle \quad \text { or } \quad\left|f_{-}\right\rangle
$$

One could have taken $\operatorname{Im}\left(p_{2}^{*} q_{2}\right)=0$ along with Eq.(5.7) to follow directly from Eq.(5.6) because $\operatorname{Im}\left(a^{*} b\right)$ and $\left(|a|^{2}-|b|^{2}\right)$ are in general independent functions of $t$. The argument involving the relative phase becomes important for the possibility (5.5). Thus, the possibility (5.3) allows $\left|f_{1}\right\rangle$ to be arbitrary, but $\left|f_{2}\right\rangle$ reduces to a CP eigenstate (see Eq.(5.8)). One can similarly show that the possibility (5.4) allows $\left|f_{2}\right\rangle$ to be arbitrary, but $\left|f_{1}\right\rangle$ reduces to a CP eigenstate. The possibility (5.5) would mean that $\left|f_{1}\right\rangle \rightarrow\left|f_{ \pm}\right\rangle$and $\left|f_{2}\right\rangle \rightarrow\left|f_{\mp}\right\rangle$, thus both basis states become CP eigenstates. This possibility has been considered in Ref. [1] in an explicit calculation using the full WWA and assuming $\left|f_{1,2}\right\rangle$ to form an orthonormal system. Then one easily sees that the three possibilities (5.3), (5.4) and (5.5) effectively merge into one, and the two basis states reduce to $\left|f_{ \pm}\right\rangle$. The present derivation shows that this last possibility is allowed but not the most general one: it is sufficient to have only one of the basis states becoming a CP eigenstate and still achieve $N_{1}=0$.

\section{CP VIOLATION DUE TO THE INTRODUCTION OF THE DECOHERENCE PARAMETER}

Starting with the initial state (2.22), let us consider the production of two final states which are CP conjugates of each other with the respective QM probabilities $P$ and $\bar{P}$. Then we can make the decomposition

$$
P=P_{\text {Furry }}+P_{\text {int }} \quad \text { and } \quad \bar{P}=\bar{P}_{\text {Furry }}+\bar{P}_{\text {int }} .
$$

The introduction of $\zeta$ replaces $P$ and $\bar{P}$ by $Q$ and $\bar{Q}$, respectively:

$$
Q=P-\zeta P_{\text {int }} \quad \text { and } \quad \bar{Q}=\bar{P}-\zeta \bar{P}_{\text {int }}
$$

giving

$$
Q-\bar{Q}=(P-\bar{P})-\zeta\left(P_{\text {int }}-\bar{P}_{\text {int }}\right)
$$


If under certain conditions $P-\bar{P}$ vanishes (e.g., Eq.(4.4)), we would get

$$
Q-\bar{Q}=-\zeta\left(P_{\mathrm{int}}-\bar{P}_{\mathrm{int}}\right) .
$$

This difference would not vanish, because $P=\bar{P}$ does not in general imply $P_{\text {int }}=\bar{P}_{\text {int }}$. Eq.(6.4) is a simple way of seeing the development of CP non-invariance proportional to $\zeta$, for any $t^{\prime}, t$. In detailed explicit calculations with the WWA for $\epsilon=-1$, this has been noticed in Refs. [1.:4. The present derivation shows the result $Q-\bar{Q} \neq 0$ due to $\zeta \neq 0$ to be general.

An interesting case arises for the like-flavoured final states $|f \otimes f\rangle$ and $|\bar{f} \otimes \bar{f}\rangle$ for $\epsilon=-1$ with $t^{\prime}=t$. Here, both $P$ and $\bar{P}$ vanish as a simple consequence of quantum mechanics. On the other hand, $Q-\bar{Q}=-\zeta\left(P_{\text {int }}-\bar{P}_{\text {int }}\right)$ need not vanish due to $\zeta$. This is merely a violation of quantum mechanics. Though one may emphasize $Q-\bar{Q} \neq 0$ as being CP violation, the important point is that due to $\zeta$ both $Q$ and $\bar{Q}$ are non-zero in general because $P_{\text {int }}$ and $\bar{P}_{\text {int }}$ need not vanish for $t^{\prime}=t$. This "CP violation" $(Q \neq \bar{Q})$ has recently been noted for $t^{\prime}=t$ in an explicit calculation within the WWA and with the further assumption $p_{S}=q_{L}=q_{S}=q_{L}$ for orthonormal basis vectors $\left|f_{1,2}\right\rangle$ [1]. The present derivation shows the simplicity and generality of $Q \neq 0$ and $\bar{Q} \neq 0$ in this context for $\epsilon=-1$ and $t^{\prime}=t$.

\section{SUMMARY}

In this paper we have derived some results concerning 2-particle correlations in the time evolution of the states $|f \otimes \bar{f}+\epsilon \bar{f} \otimes f\rangle,(\epsilon= \pm 1)$, of neutral flavoured mesons and antimesons. We have put emphasis on deriving our results independently of the Weisskopf - Wigner approximation which was used only for comparison with the general results. We have studied the role of the decoherence parameter $\zeta$, introduced as a particular way of parameterizing deviations from quantum mechanics by interpolation between QM interference $(\zeta=0)$ and complete loss of interference ( $\zeta=1$, Furry's hypothesis). Since the QM interference term depends on the basis chosen in the $(f, \bar{f})$ space, the procedure of introducing the decoherence parameter is a basis-dependent prodecure; we have tried to elucidate this point without having recourse to the specific time evolution of the WWA. In particular, we have considered the asymmetry (3.1) of unlike-flavoured and like-flavoured events as a function of the basis chosen and of the decoherence parameter. We have shown that several features, derived previously in the framework of the WWA, persist in general. An example is the proportionality of the asymmetry to $(1-\zeta)$, valid for both $\epsilon=+1$ and -1 , if we choose the basis of $\mathrm{CP}$ eigenstates and neglect $\mathrm{CP}$ violation in the amplitudes. We have derived some other related results more generally than in the literature in Sections [V]V]. 


\section{REFERENCES}

[1] R.A. Bertlmann, W. Grimus and B.C. Hiesmayr, Phys. Rev. D 60, 114032 (1999).

[2] R.A. Bertlmann and W. Grimus, Phys. Lett. B 392, 426 (1997).

[3] G.V. Dass and K.V.L. Sarma, Eur. Phys. J. C 5, 283 (1998).

[4] R.A. Bertlmann and W. Grimus, Phys. Rev. D 58, 034014 (1998).

[5] P.H. Eberhard, in The Second Daథne Physics Handbook, edited by L. Maiani, G. Pancheri and N. Paver (SIS-Pubblicazioni dei Laboratori di Frascati, Italy, 1995), Vol. I, p. 99.

[6] V. Weisskopf and E. Wigner, Z. Phys. 63, 54 (1930); ibid. 65, 18 (1930).

[7] CPLEAR Collaboration, A. Apostolakis et al., Phys. Lett. B 422, 339 (1998).

[8] W.H. Furry, Phys. Rev. 49, 393 (1936).

[9] L.A. Khalfin, Found. Phys. 27, 1549 (1997).

[10] G.V. Dass, Phys. Rev. D 60, 017501 (1999).

[11] P.H. Eberhard, Phys. Rev. Lett. 16, 150 (1966).

[12] P.K. Kabir, in Springer Tracts of Modern Physics, Vol. 52, edited by G. Höhler (Springer-Verlag Berlin, Heidelberg, New York, 1970), p. 91.

[13] G.V. Dass, Phys. Rev. D 45, 980 (1992); 49, 1672(E) (1994). 